\title{
Design of Grasping Interface for Microgrippers and Micro-Parts Used in the Microassembly of MEMS
}

\author{
Nikolai Dechev, William L. Cleghorn, James K. Mills \\ Dept. of Mechanical and Industrial Engineering, University of Toronto \\ 5 King's College Road, Toronto, Ontario, Canada, M5S 3G8 \\ dechev@mie.utoronto.ca,_cleghrn@mie.utoronto.ca, mills@mie.utoronto.ca
}

\begin{abstract}
This paper presents guidelines for designing the grasping interface between passive, surface micromachined microgrippers and micro-parts used for a microassembly system. The microassembly system is based on a robotic workstation, to which a microgripper is bonded. The workstation is capable of sequentially assembling surface micromachined micro-parts into 3D microstructures. The microgrippers and micro-parts are designed in such a way, that they each have corresponding geometrical features, which allow them to interface with each other. Previously, this work demonstrated the use of a standardized microgripper tip, and the corresponding micropart interface feature. However, there are situations when the standardized interface feature may be undesirable for a particular micro-part, or may interfere with the performance of a microstructure. Therefore, a set of new design guidelines are presented to modularize the design of the microgrippers tips and the corresponding micro-part interface features. By using the modular design, microgrippers can be tailored to grasp specific types of micro-parts, without the need for a standardized interface feature. An example of the new modular system is provided.
\end{abstract}

Index Terms: Microassembly, Microgripper, Micro-part, 3D MEMS, Microstructure.

\section{INTRODUCTION}

The purpose of a microassembly process is to create useful microsystems or microstructures, by assembling a set of micro-parts. When designing a microsystem that requires assembly, it is important to ensure that the constituent microparts are compatible with the assembly process to be used. Specifically, the constituent micro-part design is based on three main considerations, which are: (a) the function of each micro-part within the microsystem, (b) the way in which the micro-parts are joined together to create a functioning microsystem, and (c) ensuring that the micro-parts are compatible with the microassembly process used to assemble them. Note that these three considerations are ranked in relative order of importance. In other words, the functionality of the micro-part is more important than the joining mechanism, which is in turn more important than the compatibility with the microassembly process. Generally, there would be no point to assemble micro-parts with good microassembly compatibility, if this compatibility reduces the functionality of the microsystem, or inhibits the joining process.
The micro-parts used in this work are fabricated by surface micromachining, and are generally 100 to $300 \mu \mathrm{m}$ in length or width, and $5 \mu \mathrm{m}$ in thickness. Presently, the MUMPs [1] process is used to fabricate the micro-parts. The micro-parts are designed with three features to ensure that they are compatible with the microassembly process. Firstly, they have tether features [2] designed onto their sides, which allow them to be securely held and accurately located on the surface of a silicon chip. Secondly, they have a built-in joint feature [3] used to join them to other micro-parts. Thirdly, and most importantly, they have an interface feature, which allows them to be grasped by a microgripper. The microgripper tips are specifically designed to grasp the micro-part interface feature, and all microgrippers are fabricated alongside the micro-parts on the same chip. To perform a microassembly task, the microgripper is first removed from the chip by solder bonding it to the end effector of a robotic workstation [4]. Next, the microgripper is used to grasp the micro-parts and remove them from the chip, from their original fabricated locations. The robotic workstation translates and rotates the micro-parts to the target assembly site, and joins them at that site. An overview of the microassembly process is given in [5].

A number of other robotic based, serial microassembly strategies are being developed. Serial microassembly is a sequential process, in which individual assembly tasks are performed one after the other. Some examples of robotic, serial microassembly include those systems equipped with microgrippers $[6,7,8]$ or systems equipped with microtweezers [9].

\section{GRASPING INTERFACE BACKGROUND}

Ideally, a microgripper used for microassembly should be designed so that it is able to handle a wide assortment of micro-parts, of various shapes and sizes. However, designing such a versatile microgripper is challenging. Often, the design of the various micro-parts must be altered and standardized in some way, to allow the microgripper to grasp them. Alternatively, where micro-part alteration is not possible, the microgripper must be specially designed to be suitable for grasping specific types/shapes of micro-parts. In this sense, the 'grasping interface' between the micro-part and the microgripper must be considered when designing a microsystem that requires assembly. When considering the 'grasping interface', it can be designed such that it lies 
between two extreme cases. In one extreme, specific microgrippers only suitable for grasping specific micro-parts can be designed. This way, the 'grasping interface' complexity would be designed primarily into each specific microgripper, and the micro-part 'grasping interface' need not exist, or can remain relatively simple. The disadvantage to this approach is the requirement for many different microgrippers corresponding to each type of micro-part to be used. In the other extreme, a single, standard microgripper suitable for grasping all micro-parts can be designed. However, the 'grasping interface' complexity would now be designed into the micro-part designs, since they must all conform to the single standard interface feature. Regardless of how obtrusive this interface feature may be, it must be included, otherwise the micro-parts cannot be grasped.

Previously, this work employed the latter approach. A single, standard microgripper [5] was designed and various micro-parts were equipped with a corresponding standard interface feature [5]. Fig. 1(a) shows a SEM (scanning electron microscope) image of the grasping tips of the single, standard microgripper. This microgripper is a 'passive' design, in that it requires no 'active' actuation of the microgripper tips. Rather, the microgripper tips passively open and grasp a micro-part, as they are inserted into a micropart interface feature. Similarly, they passively open and release micro-parts, after those micro-parts are joined to a microstructure. Fig. 1(b) illustrates the cross-sectional view of the microgripper tips along Section A. Note that the microgripper tips have a split-level design consisting of an 'upper level' and a 'lower level'. This split-level design assists in providing a secure grasp of micro-parts, and will be described further in Section III.

Fig. 1(c) shows an SEM image of the standard interface feature located in the center/back of micro-parts. Fig. 1(d) illustrates the cross-sectional view of the interface feature along Section B. Note that the interface feature is comprised of two layers of polysilicon, denoted Poly 1 and Poly 2. The Poly 2 layer has been intentionally fabricated to create the 'raised Poly 2' structure shown in Fig. 1(d-2). The raised structure has been created using an underlying sacrificial layer of Poly 1, as described in [5]. This allows the split-level microgripper tips to 'inter-lock' with the interface feature, to create a secure grasp. Fig. 2 shows some video microscope images of various micro-parts used in this research, which are about to be grasped by the standard microgripper tips. Note that the micro-parts all have different shapes, sizes, and different joint features. However, all micro-parts are equipped with a standard interface feature, which is identical in all micro-parts, and is always located on the center/back of the micro-parts.
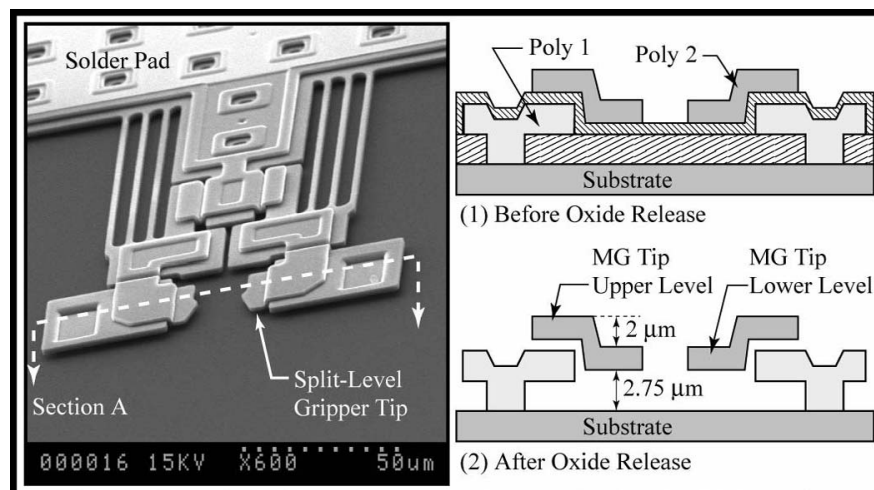

(a) SEM of Standard Microgripper

(b) Cross-Section A of Microgripper
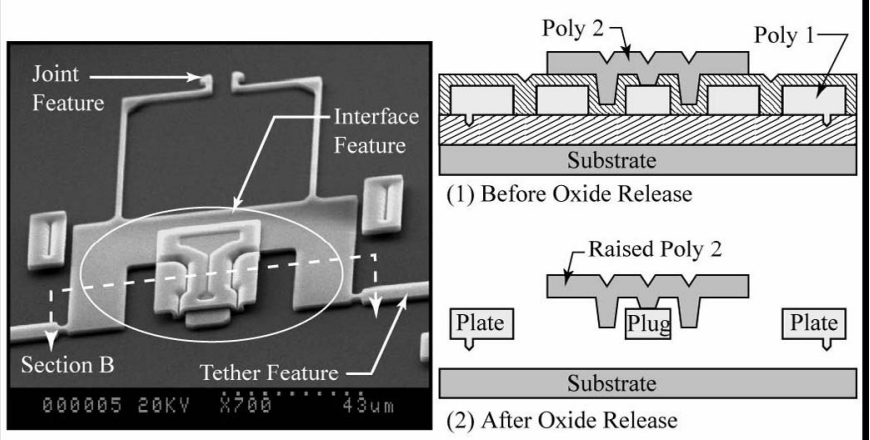

(c) SEM of Standard Interface F.

(d) Cross-Section B of Interface F.

Fig. 1 SEM Image of Microgripper Tips and Micro-Part Interface Feature With Cross-Sections of Both.
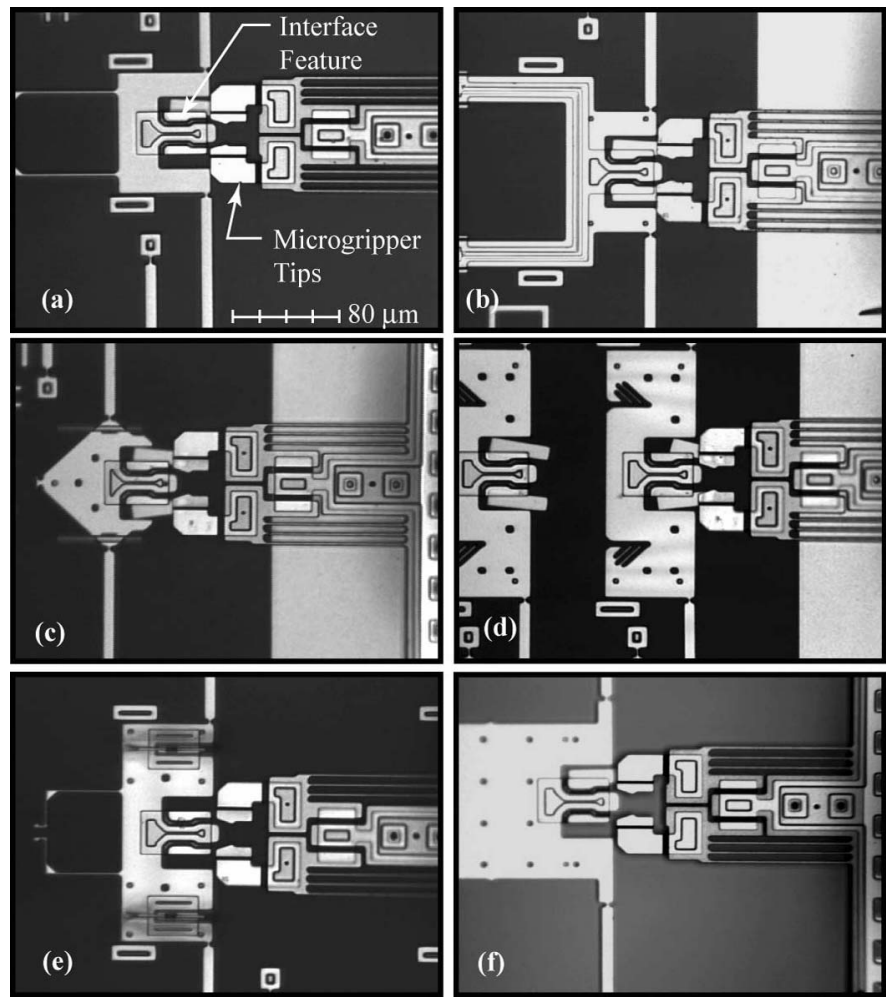

Fig. 2 Video Images of Various Micro-Parts, All using the Standard Interface Feature Design. 
Passive microgrippers are used in this work since they have a number of advantages. They do not require complex and bulky actuator designs, and do not require electrical power. Since they do not require power, they have a single bonding pad, which makes them easy to bond to the robot end effector, and makes them smaller in size than active microgripper designs. As passive designs, they employ a grasping and releasing strategy [5] that overcomes the problems of stiction, which can occur when attempting to release micro-objects with active microgrippers. As such, passive microgrippers are employed for all aspects of this microassembly work.

However, there are some disadvantages to using passive microgrippers. Passive microgrippers can only grasp and release objects that are adequately restrained, and that have specific interface feature geometries. These are reasonable requirements for micro-parts used for microassembly purposes. However, these requirements are not reasonable for general micromanipulation of non-standard, unrestrained, or irregular micro-objects, which can be better handled by active microgrippers. Additionally, the 'grasping interface' design between the microgripper and the micro-part is more complex, due to the passive grasping and release process. In comparison, active microgrippers require simple interface features such as a hole, or two parallel external sides on a micro-part.

Fig. 3 shows a sequence of video images that illustrate the process of grasping a micro-part equipped with a standard interface feature, using a passive microgripper.
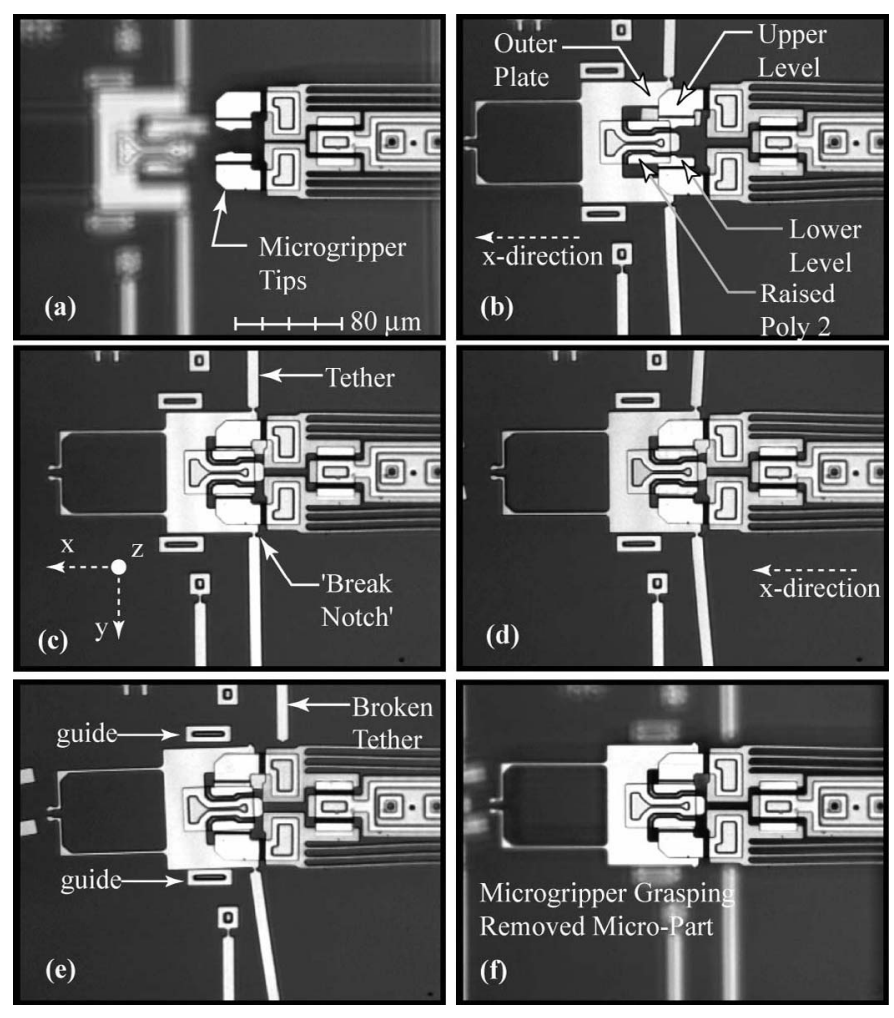

Fig. 3 Sequence of Video Images Showing the Grasp of a Micro-Part Equipped with a Standard Interface Feature, Using a Passive Microgripper.
The first step in the passive grasping process is to align the microgripper tips with the interface feature on the micropart. Fig. 3(a) shows the microgripper tips positioned approximately $30 \mu \mathrm{m}$ above the interface feature of the micropart to be grasped. The depth of focus of the microscope system is only $1.5 \mu \mathrm{m}$, and the microscope remains in-focus with the microgripper tips at all times. Therefore, all other objects either closer to, or further from the focal plane will appear out-of-focus. The field of view of these images is 320 $\mu \mathrm{m}$ horizontally by $240 \mu \mathrm{m}$ vertically. Fig. 3(b) shows the initial insertion of the microgripper tips into the interface feature. As the tips are inserted into the interface feature in the $x$-direction, they passively open outwards ( $y$-direction). Upon closer inspection of Fig. 3(b), note that lower level of the microgripper tips pass under the 'raised Poly 2 structure' (see Fig. 1(d)) of the interface feature, while the upper level of the tips pass over the 'outer plate' (see Fig. 1(d)) of the interface feature. This grasping configuration creates an interlocking geometry that restrains the micro-part in $6 \mathrm{DOF}$ (degrees of freedom). This prevents the micro-part from sliding out of the microgripper tips along the $x, y$ or $z$ axes, and prevents the micro-part from rotating out of the tips about the $x, y$ or $z$ axes. Fig. 3(c) shows the completed grasp. Note that although the micro-part is now grasped, it still remains tethered to the chip substrate.

To remove the micro-part from the chip, force is applied in the $x$-direction as shown in Fig. 3(d), to break the tethers. The deflection of the tethers can be observed in the image. The tethers have a narrow 'notch' at each end to create a stress concentration point. The tethers are designed to break at these notches when $100 \mu \mathrm{N}$ or more, is applied at the interface feature in the $x$-direction. Fig. 3(e) shows the micro-part after the first tether is broken. Continued motion in the $x$-direction will result in the break away of the second tether. Fig. 3(f) shows the released micro-part held by the microgripper approximately $30 \mu \mathrm{m}$ above the chip substrate. The micropart is now ready to be manipulated to an assembly site, and joined to another micro-part. Video movies of the grasping and tether breakaway process are available for viewing at [10]. More details on the tether design and joint process are available in [2].

\section{Design OF A MOdUlar Grasping InTERFACE}

The standard interface feature design and the corresponding microgripper design, as described in Section II, have been highly successful in past work. This combination allows a wide range of micro-parts to be securely grasped by a microgripper, during demanding operations such as tether breakaway and micro-joining. The success rate of recent tether breakaway designs is over $98 \%$ and the overall joining success rate is $89 \%$ [3]. However, the standard interface feature is not always appropriate for incorporation into some types of micro-parts. For example, it must always be located on the center/back of the micro-parts, which may interfere with their function, or may impede the assembly of other micro-parts in the region of the interface feature. Therefore, a set of new design guidelines to create a modular 'grasping 
interface' are now presented. The modular system captures the essence of the previous standard system, but can be designed onto micro-parts in other areas.

Previously, this work has been used to construct 3D micro-coils [11]. Fig. 4 shows an SEM (scanning electron microscope) image of a successfully assembled 3D micro-coil. This micro-coil consists of six individual micro-part 'loops', which are assembled together to create the complete microstructure. Due to the presence of the standard interface feature on each loop of the coil, the theoretical performance of such a micro-coil is reduced. This happens because electric capacitance can occur between adjacent interface features, as shown in Fig. 4(a). When an oscillating current passes through the coil, this capacitance effect can reduce the quality factor of the micro-coil. In other words, the standard interface features can have a negative effect on this microsystem's performance. Alternatively, it may be desirable to construct microstructures where the micro-parts must be joined at locations currently occupied by the standard interface feature. In order to remedy these situations, the interface feature must be re-designed to minimize its size/area, or re-located to another area on the micro-part.

The modular interface feature must incorporate these new requirements, yet must still employ a grasping strategy that secures micro-parts in $6 \mathrm{DOF}$, similar to the $6 \mathrm{DOF}$ grasp of the standard interface feature. It is very important to grasp micro-parts in this manner, since the forces that arise during the during the tether breakaway operation [2] and during the various micro-joining operations [3] can be relatively high, and can act in various directions. Note, at the micro-scale, stiction effects are strong and could potentially be used to stick to and hold onto micro-objects, for a 3DOF grasp using a probe tip or micro-end-effector. Although it is sometimes possible to use this strategy to attach to and hold onto unrestrained/freed micro-parts with the standard microgripper, these stiction forces are weaker than the tether breakaway forces, or the forces that occur during the micro-mechanical joining operations used in this work. Therefore, a stictiononly based grasping strategy cannot be used with this microassembly system.

To design the interface feature, three design guidelines must be used to create a 6 DOF 'grasping interface.' These are: the in-plane restraint, the out-of-plane restraint, and the rotational restraint. Fig. 5 illustrates representative geometrical designs that create these restraint conditions. The first design guideline for the 'grasping interface' is to secure the grasp in the plane of the micro-part, as illustrated in Fig. 5(a). The design consists of a 2D plug feature on the center/back of the micro-part and a corresponding feature on the microgripper. This gives the design the ability to secure the grasp in the $x$ and $y$ directions, and to prevent rotation about the $z$-axis. However, the design of Fig. 5(a) is not able to prevent translation/slip in the $z$-direction, and cannot prevent rotation about the $x$-axis or about the $y$-axis. The second design guideline is to secure the grasp in the direction that is out-of-plane with the micro-part. This is done by incorporating the features shown in Fig. 5(b) and Fig. 5(c).

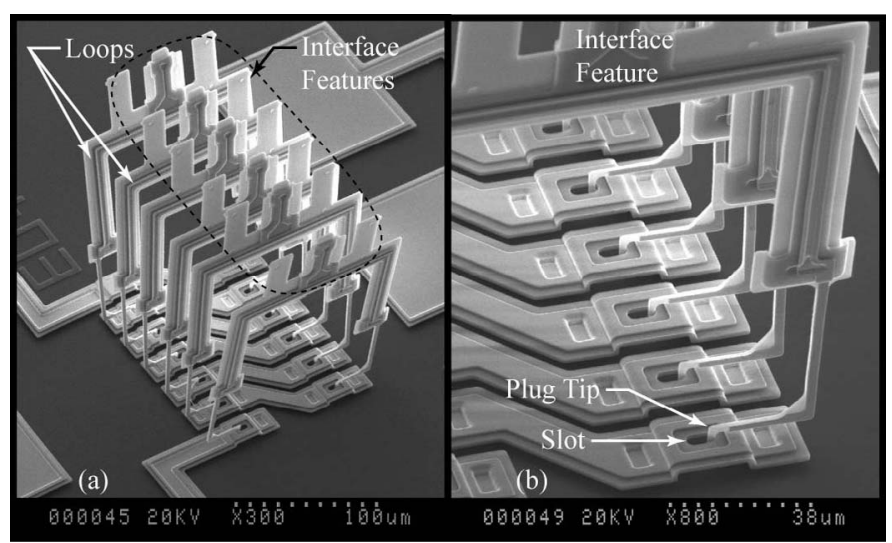

Fig. 4 SEM Image of 6-Loop Micro-coil Using Standard Interface Features on the Individual Loop Micro-Parts.

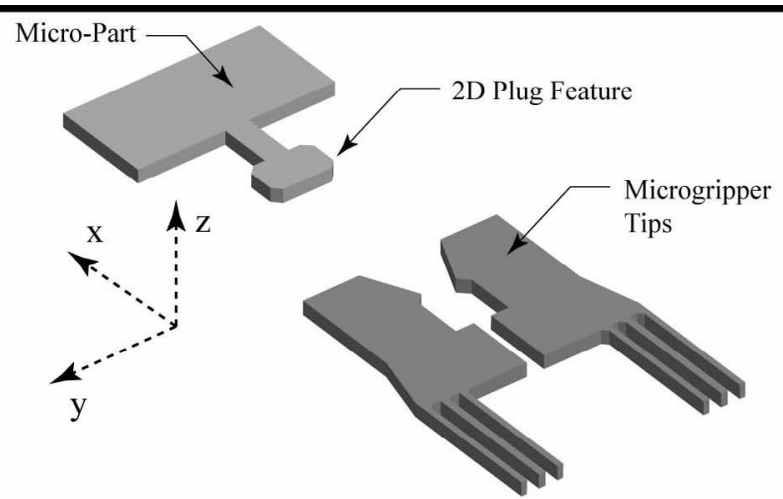

(a) Secure Grasp in the $x-y$ Plane, and about z-axis

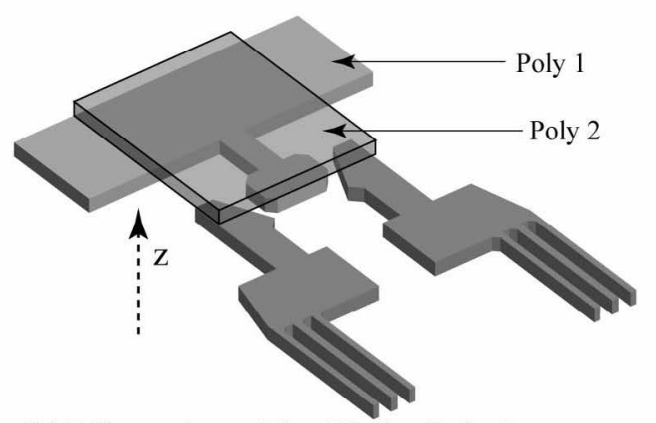

(b) Microgripper Tips Under Poly 2

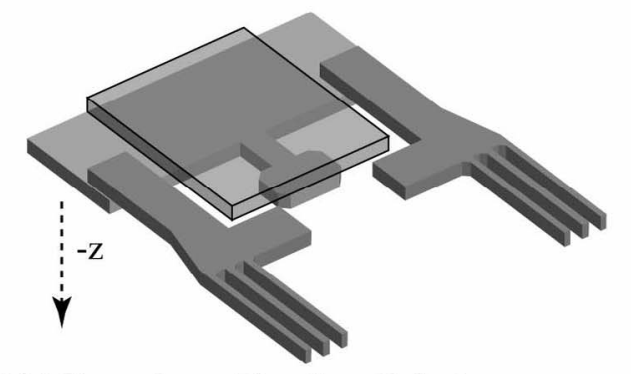

(c) Microgripper Tips Over Poly 1

Fig. 5 Illustration of Geometrical Requirements for a 6 DOF Grasping Interface. Example Shows Original Standard Design Guidelines. 
Fig. 5(b) illustrates the design used to secure the grasp in the out-of-plane direction along the $+z$-axis. This is done by designing a Poly 2 layer above the plug feature on the micropart, and ensuring that the micro-gripper tips pass under the Poly 2, hence preventing travel along the $+z$-axis. Fig. 5(c) illustrates the design used to secure the grasp in the out-ofplane direction along the $-z$-axis. This is done by designing a portion of the microgripper tips, that do not make contact with the plug feature, to pass over the Poly 1 layer of the micropart, hence preventing travel along the -z-axis. The third design guideline is to provide rotational restraint. This is done by simultaneously implementing designs (a), (b) and (c) from Fig. 5. Note that by simultaneously implementing designs (b) and (c), translation/slip along the $z$-axis is prevented, and rotation about the $y$-axis is prevented. In order to prevent rotation about the x-axis, symmetry must be employed about the plug feature, whereby each microgripper tip must be on either side of the plug feature. By applying these three design guidelines, it is possible to design microgripper tips and a micro-part interface feature that can form a secure 6 DOF grasp.

\section{IMPLEMENTATION OF MODULAR GRASPING INTERFACE}

An example microgripper and micro-part created by applying the design guidelines for the modular 'grasping interface' are now presented. Many possible variations of the microgripper and the corresponding micro-part designs can be made using these guidelines. The example presented here, as shown in Fig. 6 and Fig. 7, is just one such variation.

Fig. 6(a) shows an SEM image of a passive microgripper solder bonded to the end-effector (Metal Probe Tip) of the robotic workstation. This microgripper has grasping tips that are designed to grasp micro-parts from the outside edges, instead of the center/back as in the previous standard interface feature. Fig. 6(b) shows the details of the grasping tips. The tips of this microgripper are spaced apart by a distance $d g$, which is $140 \mu \mathrm{m}$ for this design. Distance $d g$ is arbitrary, and must match the interface feature of the corresponding micropart to be grasped.

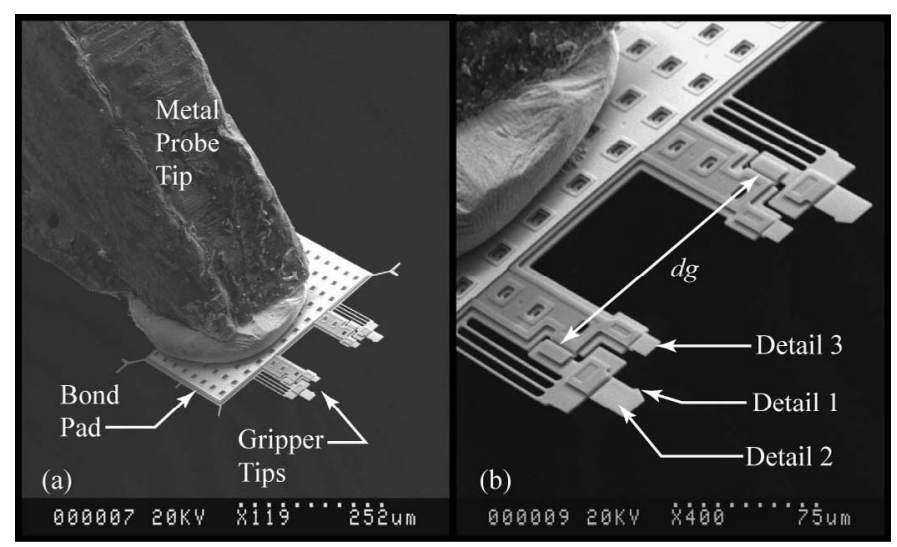

Fig. 7 SEM Image of Example Microgripper Design Incorporating Modular Grasping Interface Design

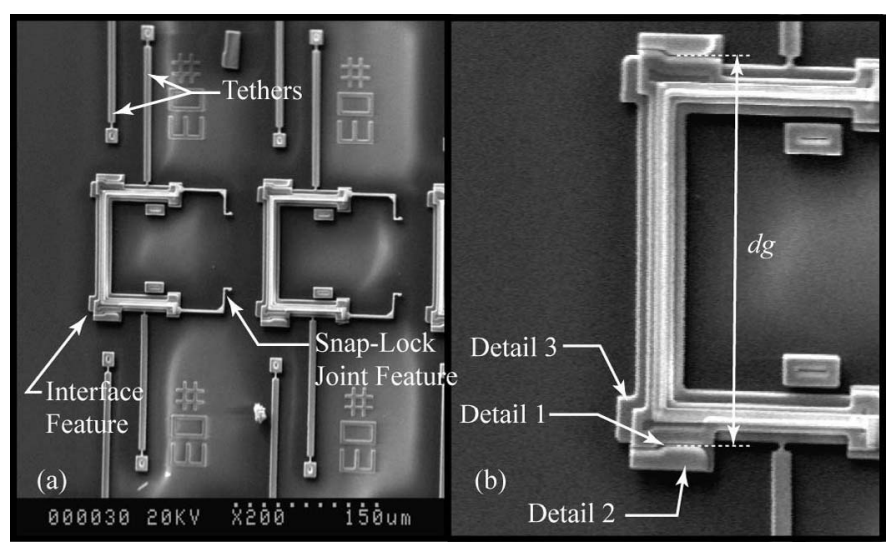

Fig. 6 SEM Image of Micro-Part Incorporating Modular Grasping Interface Design

Fig. 7(a) shows an SEM image of the micro-parts that employ the new modular interface feature. These micro-parts can be used to construct a micro-coil. Since this modular interface feature has a much smaller surface area than the previous design shown in Fig. 4(a), the quality factor of these micro-coils would be theoretically higher than previous designs. The SEM also shows the tether features and the snap-lock joint tips used for joining the micro-parts to the coil base structure. Fig. 7(b) shows the details of the modular interface feature.

Essentially, the 'grasping interface' of this design example is a version of the standard 'grasping interface' that has been split down the middle, and stretched apart by distance $d g$. Additionally, each of the gripper tips have been inverted in terms of their restraining features. By examining both Fig. 6(b) and Fig. 7(b), the design details that correspond to the modular design guidelines can be observed. Note 'Detail 1' in both figures, which corresponds to the design guideline illustrated in Fig. 5(a). This design detail is used to secure the grasp in the $x-y$ plane, and to prevent rotation about the $z$-axis. Note 'Detail 2' in both figures, which corresponds to the design guideline illustrated in Fig. 5(b). The gripper tip 'Detail 2' will pass underneath the interface feature 'Detail 2', thereby preventing slip/motion along the $+z$-axis. Note 'Detail 3 ' in both figures, which corresponds to the design guideline illustrated in Fig. 5(c). 'Detail 3' in Fig. 6(b) is part of the microgripper structure, and will pass overtop the interface feature 'Detail 3', thereby preventing slip/motion along the -zaxis. Additionally, the combination of 'Detail 2' and 'Detail 3 ' will prevent rotation about the $y$-axis and $x$-axis.

Fig. 8 shows an SEM image of a partially constructed micro-coil using the new modular 'grasping interface'. In comparison to the image shown in Fig. 4(a), it can be seen that the interface feature is much less obtrusive in this design. Additionally, it greatly reduces the surface area between adjacent loops of the micro-coil. 


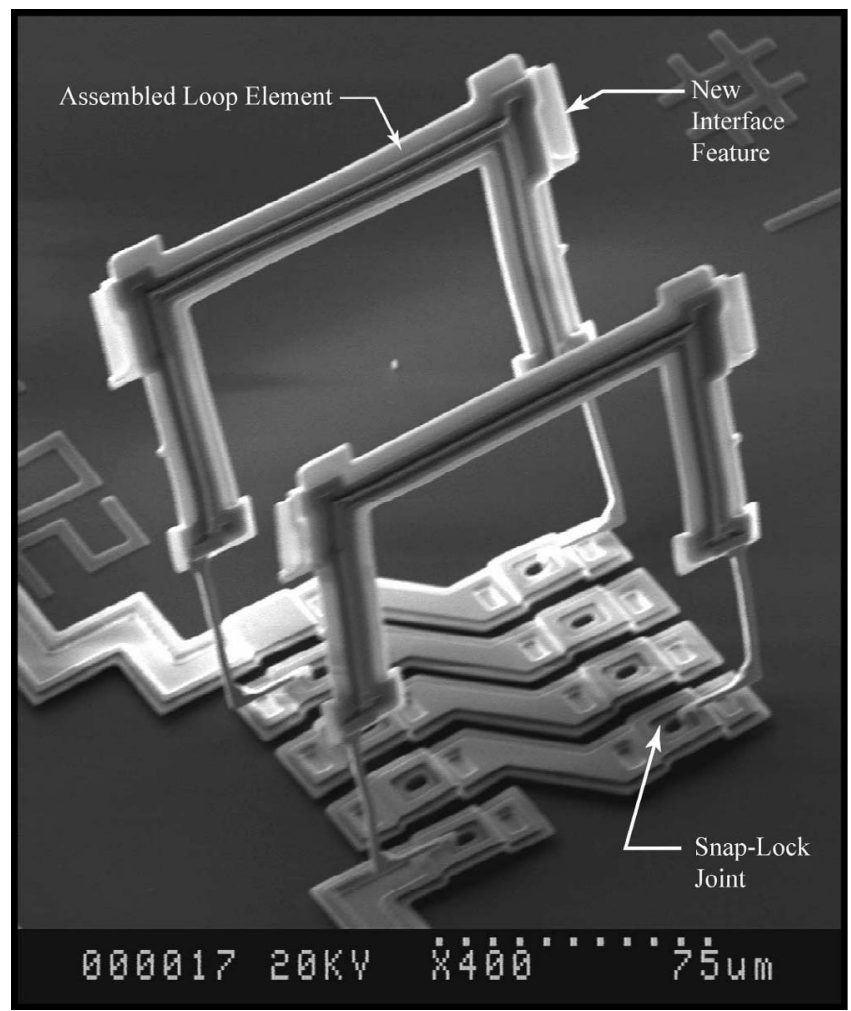

Fig. 8 SEM Image of Partially Assembled Micro-Coil using New Modular 'Grasping Interface'

\section{DISCUSSION}

By using the new modular 'grasping interface' design approach, micro-parts can be designed with less obtrusive interface features. This can be used to prevent potential reductions in microsystem functionality associated with the old 'grasping interface' design. Additionally, it can be used to create a wider assortment of microstructure designs that are

\section{REFERENCES}

[1] D. Koester, A. Cowen, R. Mahadevan, and B. Hardy, "PolyMUMPs Design Handbook Revision 9.0", MEMSCAP, MEMS Business Unit (CRONOS), Research Triangle Park, N.C., USA, 2001.

[2] N. Dechev, W. L. Cleghorn, and J. K. Mills, "Tether and Joint Design for Micro-Components used in Microassembly of 3D Microstructures", Proceedings SPIE Micromachining and Microfabrication, Photonics West 2004, San Jose, CA, Jan 25-29, 2004.

[3] N. Dechev, J. K. Mills, and W. L. Cleghorn, "Mechanical Fastener Designs for use in the Microassembly of 3D Microstructures", Proceedings ASME International Mechanical Engineering Congress and $R \& D$ Expo 2004, Anaheim, California, Nov 13-19, 2004.

[4] N. Dechev, W. L. Cleghorn, and J. K. Mills, "Microassembly of 3-D MEMS Structures Utilizing a MEMS Microgripper with a Robotic Manipulator", Proceedings of IEEE International Conference on Robotics and Automation (ICRA 2003), Taipei, Taiwan, Sept 14-19, 2003.

[5] N. Dechev, W. L. Cleghorn, and James K. Mills, "Microassembly of 3D Microstructures Using a Compliant, Passive Microgripper", Journal of Microelectromechanical Systems, vol. 13, no. 2, Apr. 2004, pp. 176-189. not impeded by the requirement of the old standard 'grasping interface' located at the center/back of a micro-part.

The example described in Section IV is one possible design using the modular 'grasping interface' out of many. Note that as long as the design guidelines are followed, the distance $d g$ used is arbitrary, and the geometries of 'Details 1 , 2 , and 3' can be changed to suit any particular application. The key with these designs is to ensure that adequate $6 \mathrm{DOF}$ restraint is achieved, using the principles shown in Fig. 5.

The modular 'grasping interface' changes the previous microassembly strategy of using a single microgripper design with a standard interface feature on all micro-parts. Now, a given microgripper design with a distance $d g$ between the tips can be used to grasp a set of specific micro-parts with a corresponding width $d g$. Therefore, for any given microassembly, a number of different microgrippers can be used to grasp a corresponding set of micro-parts. This would require changing microgrippers mid-way through an assembly process, but would lead to the benefits such as better microsystem functionality, or higher microstructure complexity.

\section{CONCLUSION}

A set of modular design guidelines that can be used for the design of the 'grasping interface' between microgrippers and micro-parts, has been described. The guidelines allow for the design of various 'grasping interfaces' that can secure micro-parts in $6 \mathrm{DOF}$, allowing them be securely held during tether breakaway and micro-joining operations. By using these guidelines, the design of the interface feature located on the micro-parts can be adjusted so that it: (a) does not reduce the micro-part/microsystem functionality, and (b) does not impede the construction complex microstructure designs. An example microstructure showing a new micro-coil design was presented to demonstrate the modular 'grasping interface'.

[6] G. Yang, J. A. Gaines, and B. J. Nelson, "A Supervisory Wafer-Level 3D Microassembly System for Hybrid MEMS Fabrications", Journal of Intelligent and Robotic Systems, vol. 37, 2003, pp. 43-68.

[7] K. Tsui, A. A. Geisberger, M. Ellis, and G. D. Skidmore, "Micromachined End-effector and Techniques for Directed MEMS Assembly", Journal Micromechanics and Microengineering, vol. 4, pp. 542-549, Jan 2004

[8] D. H. Kim, K. Y. Kim, K. Kim, J. O. Park, "Micro Manipulation System based on Teleoperation Techniques" Proceedings of the 32nd International Symposium on Robotics(ISR 2001), July 4-7, 2001.

[9] E. Shimada, J. A. Thompson, J. Yan, R. Wood, and R. S. Fearing, "Prototyping MilliRobots Using Dextrous Microassembly and Folding", Proc. ASME International Mechanical Engineering Congress and Expo (IMECE / DSCD), Orlando, Florida, Nov. 5-10, 2000.

[10] N. Dechev, "Microassembly Movie Files", [online], [cited April 1, 2005], Available from World Wide Web: http://www.mie.utoronto.ca/staff/projects/cleghorn/Microassembly/Micr oassembly-Movies/MicroassemblyMovies.html>.

[11] N. Dechev, W. L. Cleghorn, and J. K. Mills, "Construction of a 3D MEMS Microcoil Using Sequential Robotic Microassembly Operations", Proceedings ASME International Mechanical Engineering Congress and R\&D Expo 2003, Washington, D.C, Nov 15-21, 2003. 